\title{
ANNOUNCEMENT 2018 Reviewer acknowledgments
}

The Editors of Pediatric Research thank the following dedicated individuals who reviewed manuscripts in 2018.

\author{
Abad-Santos, Francisco \\ Abbass Metwalley, Kotb \\ Abend, Nicholas S. \\ Ackerman, William \\ Adami, Alexander \\ Aden, Ulrika \\ Adeyemo, Adebowale \\ Agrawal, Shipra \\ Ah Mew, Nicholas \\ Aida, Jun \\ Aikio, Outi \\ Albert, Ben \\ Albertine, Kurt \\ Ali, Mehboob \\ Alkuraya, Fowzan \\ Allegaert, Karel \\ Allison, Beth \\ Almeida, Fausto \\ Alvarez, Evelyn \\ Alvira, Cristina \\ Aly, Hany \\ Ambalavanan, Namasivayam \\ Amil-Dias, Jorge \\ Amrein, Karin \\ Anand, Vikram \\ Anastasilakis, Athanasios \\ Anderson, Peter \\ Andrews, Jennifer \\ Anikster, Yair \\ Ansong, Charles \\ Arditi, Moshe \\ Argente, Jesus \\ Arshad, Mehreen \\ Arslan, Nur \\ Arslan, Selen Serel \\ Aschner, Judy \\ Ashraf, Ambika \\ Askenazi, David \\ Assa, Amit \\ Auriti, Cinzia \\ Aydemir, $\mathrm{O}$. \\ Azab, Seham \\ Baburamani, Ana \\ Bagci, Soyhan \\ Bagga, Arvind \\ Bailey, Michael \\ Baiz, Nour \\ Bakhireva, Ludmila \\ Balamuth, Fran \\ Balmes, John R. \\ Bancalari, Eduardo \\ Bandini, Linda \\ Banerjee, Sanjay \\ Barfield, Wanda
}

Barisani, Donatella
Barkin, Shari
Baroncelli, Giampiero
Barone, Rita
Bartock, Sarah
Basu, Sriparna
Bayrakci, Umut
Bednarek, Nathalie
Bekelis, Kimon
Beken, Serdar
Belfort, Mandy
Belik, Jaques
Belkind-Gerson, Jaime
Bell, Edward
Bell, Katherine
Bel-Serrat, Silvia
Benders, Manon
Benner, Eric
Benninga, M. A.
Berg, Anne
Berger, Angelika
Berghmans, Johan
Berglund, Staffan
Bergstrom, Nancy
Bernard, Kristin
Berrington, Janet
Bhat, Ballambattu Vishnu
Bhatti, Faizah
Bhutta, Adnan
Bhutta, Zulfiqar
Bihari, Shailesh
Biniwale MD, Manoj
Bird, Stephen
Birken, Catherine S.
Bjuland, Knut Jørgen
Black, Jane
Black, Stephen
Blakely, Martin L.
Blanco, Cynthia
Blank, Douglas
Blau, Jonathan
Bloomfield, Frank
Blue, Emily
Bollati, Valentina
Bomberg, Eric
Boudry, Gaelle
Boylan, Geraldine
Boyle, Coleen
Brakeman, Paul
Brion, Luc
Brito, Alex
Britt, Rodney
Brockman, Ken
Buchhorn, Reiner

Bührer, Christoph

Burns, Jane

Burrin, Douglas

Busch, David

Cadenas-Sánchez, Cristina

Cakmak, Burcu Dincgez

Camm, Emily

Campoy, Cristina

Canani, Roberto Berni

Caridi, Gianluca

Carlo, Waldemar

Carlson, Martha

Carmichael, Suzan

Casey, Brian

Castillo-Melendez, Margie

Catov, Janet

Cederbaum, Stephen

Chalak, Lina

Chan, Marilynn

Chang, Yun Sil

Charlton, Jennifer

Charman, Tony

Chehade, $\mathrm{H}$.

Chemtob, Sylvain

Chen, Chung-Ming

Chen, JC

Chen, Xin

Chen, Zhuang-Gui

Chernikova, Diana

Cheung, Michael

Cheung, Wilson

Cheung, Po-Yin

Chin A Paw, Mai

Chock, Valerie

Choi, C

Choilero, Arnaud

Chougnet, Claire

Chouthai, Nitin

Christensen, Robert

Chromek, Milan

Chung, Paul

Clarke, Penny

Claud, Erika

Cnattingius, Sven

Coban-Akdemir, Zeynep

Cock, Charles

Coda Zabetta, Carlos

Cohen, Ronald

Cohen, Mitchell

Colan, Steven

Coles, Claire D.

Combs, Andrew

Cong, Xiaomei

Connor, Ed
Coppede, Fabio

Cornet, Marie-Coralie

Correa, Flavia

Cory-Slechta, Deborah

Costantino, Claudio

Coto, Eliecer

Cotten, Michael

Couce-Pico, Maria

Counsell, Serena

Couroucli, Xanthi

Courtoy, Pierre

Cowell, Whitney

Currie, Andrew J.

Cutfield, Wayne

Czosnyka, Marek

Dahli, Darren

Dalal, Ashwin

Dalziel, Julie

Dame, Christof

Dani, Carlo

Daniels, Stephan R

Dapaah-Siakwan, Fredrick

Davenport, Mark

David, Michael

Davidson, Joanne

Davis, Kara

Dawson, Liza

de Boode, Willem-Pieter

de Carvalho Sampaio, Helena Alves

de la Cruz, Diomel

de Meij, Tim

de Ribaupierre, Sandrine

De Vries, Linda

De Waal, Koert

Dean, Douglas

Deelchand, Dinesh

DeFreitas, Marissa

Del Pozzo, Giovanna

Delahaye, Andree

Dell, Katherine

Demmelmair, Hans

Dempsey, E. M.

Dempsey, Eugene

Deng, An-Mei

Denning, Patricia Lanee Wei

Dentz, Amelie

Deraedt, Sylvie

Derraik, Jose

Deyssenroth, Maya

Di Fiore, Juliann

Di Sieno, Laura

Diaz, Julio

Domoff, Sarah

Donner, Birgit 
Donovan, Sharon

Donovan, Brittney

Dossier, Claire

Doyle, Lex

Drehmer, Jeremy

Dreyer, Benard

Duncan, Andrea

Dupont, Christophe

Durand, Manuel

Duru, Nilgun Selcuk

Dusig, Stacey

Eder, Anne

Edlow, Andrea

Ek, Joakim

Elabiad, Mohamad T.

Elitt, Christopher

Elizur, Arnon

Embleton, Nicholas

Ene, A.

Epstein, Leon

Erdeve, Senay Savas

Escobar, Gabriel

Evans, Ronald K.

Evans, Sarah

Everson, Todd

Facciotti, Federica

Fadista, Joao

Fanaroff, Jonathan

Farrell, Ryan

Faustino, Larissa

Felderhoff-Mueser, Ursula

Feng, Mei

Fenton, Tanis

Fernandes, Ericia

Fernandez-Millan, E.

Ferreira, Raphael

Field-Ridley, Aida

Fields, David

Filipeanu, Catalin M.

Finer, Gal

Fink, Ericka L.

Finken, Martijn

Finnell, Richard

Fiocchi, Alessandro

Fisher, Karen R.

Fisher, Phil

Fitzpatrick, Emer

Fleiss, Bobbi

Fleming, Stephen

Flint, Robert

Flynn, Joseph

Fonteles, Cristiane

Forlino, Antonella

Fraser, Mhoyra

Friedman, Seth

Froehlich, Tanya

Frommer, Sarah

Frymoyer, Adam

Fucile, Sandra

Fulton, David

Fung, Camille

Gaedigk, Andrea

Galinsky, Robert

Gallentine, William

Galvez, Maida

Gano, Dawn

Garberg, Håvard

Garcia-Serrano, J. L.
Garg, Vidu

Garg, Parvesh Mohan

Garin, Eduardo

Gavilanes, Danilo

Gazzin, Silvia

Gazzolo, Diego

Gbadegesin, Rasheed

Geraghty, Patrick

Gershon, Robyn

Ghosh, Suman

Gill, Hannah

Gipson, Debbie

Gisselson, Marie

Goldfarb, David

Goldstein, Neal

Gong, Fangqi

Gonzalez, Fernando

Gonzalez-Camarena, Ramon

Gordeuk, Victor R.

Gould, Jeffrey

Gower, Adam

Grammatikopoulos, Tassos

Grange, Robert W.

Green, Thomas

Greenberg, James

Greene, Nicholas D. E.

Greenough, Anne

Greisen, Gorm

Greisen, Gorm

Gressens, Pierre

Grey, Gus

Grobman, William

Groenendaal, Floris

Grosset, Christophe

Groves, Alan

Guan, Wei-Jie

Guarino, Alfredo

Gubitosi-Klug, Rose

Gunn, Alistair

Gupta, Samir

Gyawali, C. P.

Hackam, David

Hadfield, Kristin

Hadj-Rabia, Smail

Hall, Eric

Halstead, E. Scott

Hamrick, Shannon

Hannes, Tobias

Hantos, Zoltan

Hantsoo, Liisa

Haroche, Julien

Hartel, Christoph

Hartmann, Hans

Harville, Emily

Hasab, Ali

Hasegawa, Shunji

Hawes, Katheleen

Hay, William

Hayden, John

Hazen, Rebecca

Hermann, Eugene

Hermansson, Ann

Hershkovich-Shporen, Calanit

Hibbs, AnnaMaria

Hilgendorff, Anne

Hillman, Noah

Hirst, Jonathan

Ho, Jacqueline
Hobart, Judith

Hodgson, Joshua Mark

Hoen, Anne

Hojsak, Iva

Holford, Nick

Horne, Rosemary S. C.

$\mathrm{Hu}$, Gang

$\mathrm{Hu}$, Guoqing

Huang, Pengyu

Huels, Anke

Hulzebos, Christiaan

Hüning, Britta

Hunt, Carl

Hunt, Rod

Hunter, Catherine

Hütten, Matthias

Hyde, Luke

Hynan, Michael

Ibarluzea, Jesús

Ikeda, Kazuyuki

Im, Kiho

Ingulli, Elizabeth

Iorio, Raffaele

Irie, Ryusuke

Iroh Tam, Pui-Ying

Islam, A.

Ismail, Eman

Iwashima, Satoru

Jaeken, Jaak

Jain, Amish

Jain, Deepak

Jakobsen, Lotte

Jantzie, Lauren

Jasarevic, Eldin

Jefferies, John L.

Jellema, Reint

Jeng, Michael

Jensen, Erik A.

Jeon, You Hoon

Jerjes-Sanchez, Carlos

Ji, Hong

Jiang, Zedong

Jing, Hongwo

Johnson, Meshell

Johnstone, Edward

Josephsen, Cassandra

Julian, Megan

Jusko, Todd

Juul, Sandra

Kaciroti, Niko

Kajantie, Eero

Kallapur, Suhas G.

Kamdar, Aditi

Kamijo, Keita

Kandasamy, Jegen

Kapan, Julie

Kaplan, Bernard S.

Karahan, Gonca E.

Karakuş, Osman

Kardon, Gabrielle

Kaskel, Frederick

Kato, Tsuguhiko

Kaufman, David

Keijzer, Richard

Kelleher, Kelly

Kelleher, Meredith

Kelly, Claire

Kemp, Matthew
Kernohan, Kristin

Keszler, Martin

Khalil, Markus

Khashan, Ali

Khashana, Abdelmoneim

Kim, Kwang

Kim, Jee Hyun

Kimmel, Mary

Kirby, Russell

Kishimoto, Jessica

Klaassen, Sabine

Klebanoff, Mark

Klebermass-Schrehof, Katrin

Klungsøyr, Kari

Kochi, Cristiane

Kogon, Amy

Kogut, Katherine

Kohli, Rohit

Kolacek, Sanja

Kolísko, Martin

Kondo, Yutaka

Konduri, Girija

Kong, Kai Ling

Kong, Sek Won

Kooi, Elisabeth

Kourembanas, Stella

Kramer, Boris

Kribs, Angela

Krishnan, Usha

Kuehn, Devon

Kuja-Halkola, Ralf

Kumar, Ashok

Kurepa, D

Kussmann, Martin

Kutney, Katherine

Kwong, Kenny

La Russa, Daniele

LaHaye, Stephanie

Lakshminrusimha, Satyan

Lal, Charitharth

Lam, Christina

Lamb, Fred

Lande, Marc

Lang, Pierre-Olivier

Langer, Thomas

Lapidus, Nathanael

Lapointe, Anie

Laptook, Abbot

Lasky-Su, Jessica

Lau, Chantal

Lau, Christopher

Laughon, Matthew

Lavine, Joel E.

Lawson, Louise

Lee, Jennifer

Lee, Jung Eun

Leeman, Kristen

Leeson, Paul

Lei, Wei-Te

Lei, Yunping

Lemley, Kevin

Lenartowicz, Malgorzata

Lennartsson, Finn

Leung, Ting Fan

Leuzzi, Vincenzo

Leviton, Alan

Levy, Philip

$\mathrm{Li}$, Chengwen 
Li, Jian

Li, Marilyn

Li, Shengxu

Liang, Ma

Licht, Daniel

Liedel, Jennifer

Lien, Reyin

Lin, Ming

Lin, Simon

Lingappan, Krithika

Lipstein, Ellen

Lisonkova, Sarka

Liu, Jia-Ren

Locatelli, Paola

Lodha, Abhay

Lodish, Maya

Longoni, Mauro

Looman, Wendy S.

Lopez Gutierrez, Juan Carlos

Lorenz, Laila

Lougaris, Vassilios

Lu, Jing

Luban, NLC

Lucendo, Alfredo J.

Lumeng, Julie

Luyckx, Valerie

Lwin, Su

Maahs, David

MacLean, J. E.

Maffeis, Claudio

Magdalena, Budisteanu

Maggio, Luca

Maheshwari, Akhil

Majnik, Amber V.

Malaeb, Shadi

Malhotra, Atul

Malik, Rohan

Mallard, Carina

Mandell, Erica

Mannisto, Tuija

Marchetto, Carol

Marchisio, Paola

Marini, Carla

Mark, Peter J.

Markel, Troy

Martens, Dries S.

Martin, Camilia

Martin, Lee

Martinelli, Massimo

Martinez Biarge, Miriam

Martinez-Vizcaíno, Vicente

Martini, Silvia

Mashima, Ryuichi

Masliukov, Petr

Mason, Rebecca

Massaro, An

Massella, Laura

Mathur, Amit

Matsell, Douglas

Matson, Adam

Matsuda, Tadashi

Matthijs, gert

Matute, Juan

Maxwell, Jessie

McCausker, Christine

McClain, Kenneth

McDonald, Teresa

McElroy, Steven
McGrath, Jacqueline

McNally, James

Medeiros, Liciane

Meier, Paula

Melnik, Bodo

Mendelsohn, Alan

Mersha, Tesfaye

Meyer, Haakon E.

Mietzsch, Ulrike

Miletin, Jan

Miller, Bradley

Miller, Steven

Miller, Suzanne

Miller, Marcus

Millis, J. Michael

Mills, David

Minamisawa, Susumu

Miozzo, Monica

Mirmiran, Parvin

Mishra, Om P.

Mitchell, Laura

Mitchell, Wendy

Mitra, Subhabrata

Mo, X-M

Mock, Donald

Mok, Elise

Momb, Jessica

Montini, Giovanni

Moodley, Manikum

Moody, Emily

Moore, Holly

Moore, James

Morag, Iris

Morelli, Lorenzo

Morello, Roy

Morowitz, Michael

Morrison, Janna

Morty, Rory

Moscona, Anne

Moss, Timothy

Motawea, Hanaa

Mpabalwani, Evans M.

Muensterer, Oliver J.

Muir, Amanda

Mulraney, Melissa

Muniraman, Hemananda

Munzer, Tiffany

Muraca, Giulia

Muraji, Toshihiro

Muro, Andres

Murphy, Susan

Murray, Deirdre

Muszynski, Jennifer

Nair, Syam

Nambiar, Smita

Nandi, Deipanjan

Nanthakumar, N. Nanda

Narayan, lyer

Natarajan, Girija

Nathan, Brandon

Naulaers, Gunnar

Naulaers, Gunnar

Nelson, Charles

Nemer, Georges

Nemeth, Elizabeta

Neu, Josef

Newman, Nicholas

$\mathrm{Ng}$, Derek
Niel, Olivier

Nielsen, Heber

Nigam, Vishal

Noori, Shahab

Northington, Frances

Nosarti, Chiara

Novotny, Edward

Nugent, Alan

Numis, Adam

Nuolivirta, Kirsi

Oberhoffer, Renate

Oberle, Megan

Odeniyi, Fisayo

O'Donnell, Martha

Oei, Ju Lee

Ohls, Robin

Okun, Jürgen

Oliveira, Eduardo

Oliver, Emily

Olson, Timothy

Olusanya, Bolajoko

Olutoye, Oluyinka

Ong, Ken

Onland, Wes

Onouchi, Yoshihiro

OReilly, Michael

O'Reilly, Michelle

Orel, Rok

O'Rourke, Ashli

O'Sullivan, Justin

Owen, Leah A.

Pammi, Mohan

Panayotacopoulou, Maria

Paneth, Nigel

Papanna, Ramesha

Parikh, Nehal

Parikh, Nisha

Parikh, Pavan

Park, Sung Gyoo

Parker, Margaret

Pastores, Gregory

Patel, Ravi

Patel, Aloka

Patel, Neil

Paternina, Angel

Patrick, Stephen

Paul, Ian

Paula-Ribeiro, Marcelle

Paulsen, Megan

Pearlman, S

Perrella, Sharon

Pesch, Megan

Peterson, Courtney

Petito, Lucia

Picarsic, Jennifer

Pierangeli, Alessandra

Pierro, Agostino

Pillekamp, Frank

Pillow, Jane

Poets, Christian

Polaner, David

Polglase, Graeme

Polgreen, Lynda

Polites, Stephanie F.

Porges, Stephen

Porter, Jr., George

Prabhakaran, Sandhya

Pranzatelli, Michael R.
Prasad, Kailash

Prawitt, Dirk

Pressler, Ronit

Prohaska, Joseph

Provenzi, Livio

Pruna, Dario

Puopolo, Karen

Purdue-Smith, Alexandra

Puri, Prem

Querfeld, Uwe

Quintana, Penelope

Quinzil, catarina

Raffay, Thomas

Raimondi, Francesco

Rajamma, Usha

Ramakrishnaiah, Raghu

Ramanathan, Rangasamy

Ramani, Manimaran

Ramirez-Velez, Robinson

Randhawa, Inderpal

Rao, Rakesh

Raol, Yogendra H.

Rasmussen, Lisa

Ratliff, Brian

Razmus, Ivy

Re, Rebecca

Redline, Raymond

Regier, Debra

Reichman, Nancy E.

Reigart, Routt

Rennie, Donna C.

Resch, Bernhard

Reynolds, Clare

Reynolds, Rebecca M.

Rheault, Michelle

Ricci, Zaccaria

Richard, Melissa

Rieder, Michael

Rincon, Jaimar

Riordan, Sean

Rivkin, Michael

Robel-Tillig, Eva

Roberts, Calum

Roberts, James

Robertson, Nicola

Robinson, Daniel

Rocha-Ferreira, Eridan

Rocque, Brandon A.

Rodig, Nancy

Rodríguez, Alina

Rodriguez, Ana

Rodriguez, Gerardo

Rodriguez-Fanjul, J

Rogers, Lynette

Rogers, Elizabeth

Rojanasarot, Sirikan

Romero, Roberto

Rose, Sean

Ross, Michael

Ross, Kristie

Rosser, Franziska

Rothblum-Oviatt, Cynthia

Rotteveel, Joost

Rozance, Paul

Rumpf, Wolfgang

Ryan, Patrick

Ryder, Justin

Sabatini, Colleen 
918

Sabir, Hemmen

Sacchetti, Lucia

Said, Mariam

Saito, Hiroshi

Sakowicz, Agata

Salafia, Carrie

Salas, Ariel

Salo, Eeva

Salvatore, Silvia

Samelson-Jones, Ben

Sampath, Venkatesh

Sanders, Robert

Saneto, Russel P

Sankar, Raman

Santorelli, Filippo

Santos, Fernando

Santos, Rute

Santos, Susana

Sato, Sakura

Saugstad, Ola

Scala, Melissa

Scharf, Rebecca

Scheible, Kristin

Schiff, Manuel

Schiffmann, Raphael

Schmid, Manuel

Schmolzer, Georg

Schneider, Michael

Schooling, Catherine

Schulzke, Sven M.

Schwartz, Betsy

Scott, Rodney

Sebire, Guillaume

Seethapathy, Jayashree

Seidman, Michael

Selkie, Ellen

Sferra, Thomas J.

Shah, Prakeshkumar

Shah, Dilip

Shah, Prachi

Shah, Sejal

Shah, Shetal

Shah, Vibhuti

Shaikh, Nader

Shank, Erik

Shankaran, Seetha

Shapiro, Steven

Shaw, Gary

Sheldon, Candice

Shellhaas, Renee

Sheppard, Kelly

Shepperd, Sarah

Sherman, Philip

Sherr, Elliott

Shimada, $\mathrm{H}$.

Shinnar, Shlomo

Shivanna, Binoy

Siddiqi, Kamran

Sieverding, Ludger
Sifrim, Daniel

Sigman, Aric

Silverstein, Faye

Simmer, Karen

Simmons, Rebecca

Simoes-e-Silva, Ana Christina

Simon, Dennis

Singer, Lynn

Sinha, Aditi

Skiold, Beatrice

Slack, Michael

Slaughter, Jonathan

Smith, Phillip

Smith, A David

Smith, Gordon

Smolich, Joesph

Snowden, Jonathan

Snyder, Evan

Socha, Piotr

Sola-Visner, Martha

Solberg, Rønnaug

Sood, Akshay

Spagnuolo, Maria Immacolata

Spong, Catherine

Stanworth, Simon

Stark, Michael

Stenson, Ben J.

Stevenson, David

Stewart, Christopher J.

Stewart, Rosalyn W.

Stojanović, Vesna

Stonestreet, Barbara

Storme, Laurent

Stroustrup, Annemarie

Su, Yong

Sun, Jin

Sundel, Robert

Taglauer, Elizabeth

Taitz, Jonny

Takahashi, K.

Talavera, Maria

Talge, Nicole

Tan, Cin Cin

Tan, Jason K. G.

Tannous, Paul

Tasiang, Gregory

Tauber, Kate

Tea, Illa

Ten, Vadim

Teramo, Kari

Teresa Munoz-Quezada, Maria

Tezuka, Junichiro

Thadhani, Ravi

Thayyil, Sudhin

Thomas, Diana

Thompson, Deanne

Thompson, Mike

Thomsen, Kate

Thongprayoon, Charat
Torday, John

Trachtman, Howard

Tremoulet, Adriana

Triggiani, Massimo

Troost, Jonathan

Tryggestad, Jeanie

Tsuprun, Vladimir

Tullius, Kjell

Tulloh, Robert

Turan, Sifa

Turner, Mark

Tymofiyeva, Olga

Uday, Suma

Uli, Naveen

Umano, Giuseppina Rosaria

Underwood, Natasha

Urrestarazu, Elena

Ursini, Matilde Valeria

Vajro, Pietro

Vali, Payam

Van Bel, Frank

van de Looij, Yohan

van den Anker, Johannes

van der Burg, Jelske

Van Elburg, Ruurd

van Vonderen, Jeroen J.

van Wijngaarden, Edwin

Vanhatalo, Sampsa

Vannucci, Susan

Vargas-Poussou, Rosa

Varisco, Brian M.

Vasconcelos, Mariana

Venkatesh, Sudhakar K.

Vento, Maximo

Verma, Sanjay

Verrotti, Alberto

Versteegh, Florens

Vianna, Lauro

Vickers, Mark

Victor, Suresh

Vidal, Josep

Viemann, Dorothee

Vigevano, Federico

Vilanova Sanchez, Alejandra

Vincent, Jean-Louis

Viswanathan, Anuradha

Vitale, Serena

Vitek, Libor

Vitiello, Peter

Vogelezang, Suzanne

Vora, Neeta

Voskuijl, Wieger

Wade, Terrance

Walton, Sarah

Wang, Lei

Wang, Xinglong

Ward, Robert

Warner, Barbara

Wassef, Michel
Wassink, Guido

Watterberg, Kristi

Weber, Lutz

Weisz, Dany

Weitkamp, Joern-Hendrik

Welch, Martha G.

Wells, M

Wennberg, Richard

White, Robert

Widhalm, Kurt

Wikstrom, J.

Wiley, Luke

Williams, Cathy

Wilson, Karen

Wilson-Costello, Deanne

Wisnowski, Jessica

Witchel, Selma

Wlodek, Mary

Wojcik, Monica

Wolff, Jason

Wong, Cynthia

Wood, Charles

Woods, Amina S.

Woolf, Alan

Wright, lyde

Wright, Nicola

Wright, Robert

Wurzner, Reinhard

Wusthoff, Courtney

Wynn, James

Xie, Linglin

Yamada, Yosuke

Yan, Biao

Yang, Sam

Yates, Andrew

Yawno, Tamara

Yeargin-Allsopp, Marshalyn

Yeh, Ann

Yeh, Tsu Fuh

Ying, Gui-shuang

Yorgin, Peter

Yost, Christian

Young, Karen

Younge, Noelle

Zachariassen, Gitte

Zamora, Tara

Zaniqueli, Divanei

Zeidan, Haidy

Zemel, Babette S.

Zerr, Danielle

Zhang, Jun

Zhang, Bing

Zickafoose, Joseph S.

Zinkhan, Erin

Zoja, Carlamaria 